\title{
Spin-dependent Pomeron and Odderon in elastic proton-proton scattering
}

\author{
Yoshikazu Hagiwara $^{1}$, Yoshitaka Hatta ${ }^{2, a}$, Roman Pasechnik ${ }^{3}$, Jian Zhou ${ }^{1}$ \\ ${ }^{1}$ Key Laboratory of Particle Physics and Particle Irradiation (MOE), Institute of Frontier and Interdisciplinary Science, Shandong University \\ (QingDao), Shandong 266237, China \\ 2 Physics Department, Brookhaven National Laboratory, Upton, NY 11973, USA \\ ${ }^{3}$ Department of Astronomy and Theoretical Physics, Lund University, 22100 Lund, Sweden
}

Received: 11 March 2020 / Accepted: 2 May 2020 / Published online: 15 May 2020

(C) The Author(s) 2020

\begin{abstract}
We introduce a new model of near-forward elastic proton-(anti)proton scattering at high energy based on the modern formulation of Pomeron and Odderon in terms of Wilson lines and generalized transverse momentum dependent distributions. We compute the helicity-dependent elastic amplitudes $\phi_{1,2,3,4,5}$ in this model and study their energy dependence from the nonlinear small $-x$ evolution equations. While both Pomeron and Odderon contribute to helicity-flip processes in general, in the forward limit $t=0$ only the double helicity-flip amplitude $\phi_{2}$, dominated by the spindependent Odderon, survives. This may affect the extraction of the $\rho$ parameter as well as the total cross section in the LHC energy domain and beyond.
\end{abstract}

\section{Introduction}

The elastic proton-(anti)proton scattering at high energies becomes an important source of information about the multilayer proton structure [1]. While the gluon-driven exchanges are dominant at asymptotically high energies (small- $x$ ), an elastic scattering implies, at least, a (colour-singlet) pair of correlated gluons propagating in the $t$-channel known as the QCD Pomeron (see e.g. Ref. [2] and references therein), in analogy to the leading pole exchange with Regge trajectory of the highest intercept [3] (for more detailed on the Regge theory, see Ref. [4]). Even larger numbers of interacting gluons can be exchanged in an elastic scattering process, but the role of such multi-gluon interactions in elastic scattering yet remains uncertain, particularly, from the QCD point of view.

An odd-number gluon exchange starting from the leading triple-gluon one corresponds to the crossing-odd Odderon contribution in the Regge picture [5,6] (see also Ref. [7]). It

a e-mail: yhatta@bnl.gov (corresponding author) was proposed back in the 70s in Ref. [8] that the Odderon contribution may be non-negligible compared to that of the Pomeron in the high-energy limit. However, while an experimental observation of the Odderon is yet unavailable, an exact magnitude and characteristics of such an elusive effect from theoretical viewpoint remain largely unknown and are the subjects of an intense debate and even controversial statements in the literature.

The recent outbreak of Odderon activity (see e.g. Refs. [911]) is largely triggered by the precision TOTEM data at the highest energy of the LHC, $\sqrt{s}=13 \mathrm{TeV}$, on total $\sigma_{\text {tot }}$ [12] and differential $d \sigma / d t$ [13] $p p$ cross sections, as well as on the real-to-imaginary ratio of the elastic nuclear amplitude at the optical point, the so-called $\rho$-parameter [14]. Introducing the total helicity non-flip elastic amplitude $T(s, t)$ as a function of the total c.m. energy squared $s$ and four-momentum transfer squared $t$, the basic measurable quantities of the elastic scattering read

$$
\frac{d \sigma}{d t}=\frac{\left(1+\rho(s, t)^{2}\right)}{16 \pi s\left(s-4 M^{2}\right)}(\operatorname{Im} T(s, t))^{2}, \quad \rho(s, t)=\frac{\operatorname{Re} T(s, t)}{\operatorname{Im} T(s, t)}
$$

so that the $\rho$-parameter is related to the total and differential (at vanishing momentum transfer) cross sections as follows

$$
\begin{aligned}
& \left.\frac{d \sigma}{d t}\right|_{t=0}=\frac{1+\rho^{2}}{16 \pi} \sigma_{\text {tot }}^{2}, \quad \sigma_{\text {tot }}=\frac{\operatorname{Im} T(s, t=0)}{\sqrt{s} \sqrt{s-4 M^{2}}}, \\
& \rho \equiv \rho(s, t=0)
\end{aligned}
$$

where $M$ is the proton mass. The $\rho$-parameter is small at TeV energies, $\rho \sim 0.1$, and has been extracted by the TOTEM collaboration in Ref. [14] from the experimental data on $d \sigma / d t$ near $t \approx 0$ using the Coulomb-Nuclear Interference (CNI). As long as $\rho(s)$ is known with sufficiently high precision, Eq. (2) is used to determine $\sigma_{\text {tot }}(s)$. The dominating claim is 
that a growth of the total cross section with energy, together with a decreasing $\rho$-parameter, as well as a qualitative difference of differential cross sections of $p p$ and $p \bar{p}$ collisions [15-17], all are associated with the Odderon effect. There are some concerns in the literature, however, about the validity of the experimental procedure of $\rho$ extraction (see e.g. $[18,19]$ ) and to the Odderon interpretation of its decrease with energy (see e.g. Refs. [11,20]), and hence more care is needed to justify the magnitude and the significance of the Odderon effect in the $\rho$ measurement. In off-forward kinematics, a substantiated claim about the Odderon effect and its significance is made recently from the shape analysis of the elastic differential $p p$ and $p \bar{p}$ cross sections based upon their scaling properties in Ref. [21]. In the current study, we instead consider a possible Odderon effect and its energy dependence at the optical point of vanishing $t \approx 0$ only and leave the analysis of $t$-dependence for a future work.

The usual rationale, similarly to the Pomeron, is that the Odderon is assumed to not flip the helicities of the scattered hadrons. Indeed, the existing theoretical formulations and the procedure of $\rho$ extraction from the experimental data itself heavily rely on the presumption about an absence or a large suppression of helicity-flip processes at high energies. In this work, we question this convention and, in particular, explore a viable possibility that the helicity-flip elastic amplitude may be non-negligible at high energies. To our knowledge, this has neither been confirmed nor disproved by direct experimental measurements in the $\mathrm{TeV}$ region. On the other hand, it has been suggested in the literature that the Odderon can contribute to helicity-flip amplitudes [22-25]. Yet, the exact treatment of the problem has been difficult due to the lack of a systematic way to connect the Pomeron and Odderon with the spin degrees of freedom of the scattering (composite) particles such as protons in QCD. ${ }^{1}$

Recently, however, there has been a significant progress in our understanding of the interplay between the Odderon and the proton spin [29-34]. In the Deep Inelastic Scattering (DIS) at small- $x$, the Color Glass Condensate (CGC) framework [35] provides a consistent description of the Pomeron and Odderon in terms of Wilson line correlators. Their couplings with various proton polarization states can be completely parametrized by the generalized transverse momentum dependent distributions (GTMDs) [34]. Indeed, the gluon Sivers function [36] at small- $x$ is connected to the Odderon in the forward limit [29-31] and participates in the proton helicity-flip reactions including the unpolarised elastic scattering processes. In particular, it has been observed that the so-called spin-dependent Odderon [29] can flip the proton helicity even in the forward limit, and this effect can

\footnotetext{
${ }^{1}$ For an earlier discussion of the Pomeron helicity flip observables for phenomenological scalar, vector and tensor Pomeron-proton couplings, see Ref. [26], and also, Refs. [27,28].
}

survive at high energies since the Odderon intercept is exactly equal to unity [7].

Motivated by these developments, in this paper we introduce a new model of near-forward elastic proton-proton scattering designed for the $\mathrm{TeV}$ region and beyond. By treating one of the protons within the quark-diquark model, we can devise a setup analogous to DIS in the so-called dipole frame. In this frame, helicity-flip amplitudes can be calculated by exchanging the spin-dependent Pomeron and Odderon. We then study their energy dependence at $t=0$ by numerically solving the small- $x$ evolution equations for Pomeron and Odderon. Of course, in near-forward $p p$ scattering there is no apparent hard scale (like the photon virtuality $Q^{2}$ in DIS) which guarantees the use of perturbative approaches. However, in the $\mathrm{TeV}$ region one can consider the saturation momentum $Q_{s}$ as a dynamically generated hard scale.

The paper is organised as follows. In Sect. 2, we introduce the basic helicity amplitudes of the elastic $p p$ scattering and discuss their role at high energies. In Sect. 3, we derive the helicity amplitudes in the quark-diquark dipole model and discuss their main properties. In Sect. 4, energy dependence of the helicity amplitudes and their ratios is numerically studied from the nonlinear small- $x$ evolution equations. Finally, a brief summary and concluding remarks are given in Sect. 5 .

\section{Helicity amplitudes}

Consider near-forward proton-proton elastic scattering $P_{1} P_{2} \rightarrow P_{1}^{\prime} P_{2}^{\prime}$ at high energies schematically shown in Fig. 1, with 4-momenta satisfying

$$
\begin{aligned}
& P_{1}^{\mu} \approx \delta_{+}^{\mu} P_{1}^{+}, \quad P_{2}^{\mu} \approx \delta_{-}^{\mu} P_{2}^{-}, \\
& \Delta^{\mu}=P_{1}^{\prime \mu}-P_{1}^{\mu}=P_{2}^{\mu}-P_{2}^{\prime \mu} \approx \delta_{i}^{\mu} \Delta_{\perp}^{i},
\end{aligned}
$$

where $i=1,2$ denotes the transverse momentum components.

We introduce the spin-dependent elastic amplitudes $\left\langle\lambda_{1}^{\prime} \lambda_{2}^{\prime}|T| \lambda_{1} \lambda_{2}\right\rangle$ [37-40] where $\lambda=2 h= \pm 1$ represents the helicity of each proton (multiplied by two, for convenience). These helicity amplitudes depend on $s \approx 2 P_{1}^{+} P_{2}^{-}, t \approx-\Delta_{\perp}^{2}$ as well as the azimuthal angle $\varphi=\operatorname{Arg}\left(\Delta_{\perp}^{1}+i \Delta_{\perp}^{2}\right)$. We factor out the $\varphi$-dependence as ${ }^{2}$

$$
\left\langle\lambda_{1}^{\prime} \lambda_{2}^{\prime}|T| \lambda_{1} \lambda_{2}\right\rangle \equiv e^{\frac{i}{2}\left(\lambda_{1}-\lambda_{2}-\lambda_{1}^{\prime}+\lambda_{2}^{\prime}\right) \varphi}\left\langle\lambda_{1}^{\prime} \lambda_{2}^{\prime}|\tilde{T}| \lambda_{1} \lambda_{2}\right\rangle
$$

and switch to the commonly used notation

$$
\begin{aligned}
& 8 \pi \phi_{1}(s, t)=\langle++|\tilde{T}|++\rangle, \\
& 8 \pi \phi_{2}(s, t)=\langle++|\tilde{T}|--\rangle,
\end{aligned}
$$

\footnotetext{
$\overline{2}$ The exact phase factor depends on one's convention when defining the nucleon spinors, and the one we adopt here may differ from those in the literature. Of course, the overall phase is unobservable and physically unimportant.
} 


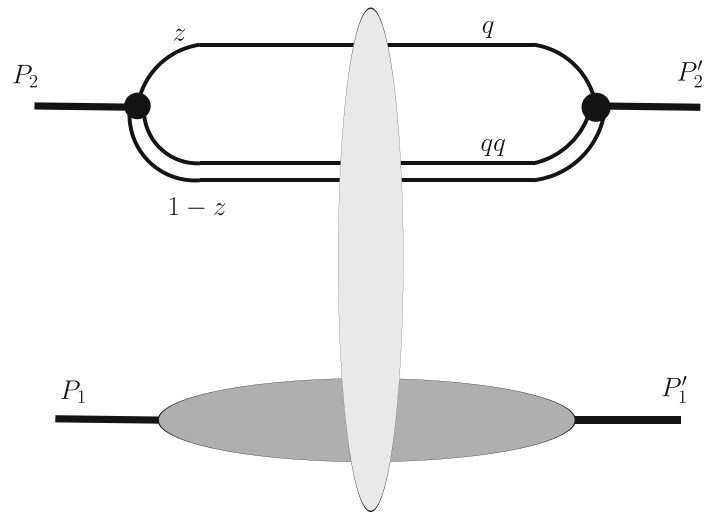

Fig. 1 Schematic illustration of the light-front dipole picture of elastic $p p$ scattering driven by a quark-diquark dipole scattering off a proton target by means of the Pomeron and Odderon exchanges in the $t$-channel commonly denoted by a vertical grey blob

$$
\begin{aligned}
& 8 \pi \phi_{3}(s, t)=\langle+-|\tilde{T}|+-\rangle, \\
& 8 \pi \phi_{4}(s, t)=\langle+-|\tilde{T}|-+\rangle, \\
& 8 \pi \phi_{5}(s, t)=\langle++|\tilde{T}|+-\rangle .
\end{aligned}
$$

$\phi_{1,3}$ are the helicity non-flip amplitudes, $\phi_{2,4}$ are the double helicity-flip amplitudes and $\phi_{5}$ is the single helicity-flip amplitude. They are normalized such that the elastic differential cross section reads

$$
\frac{d \sigma}{d t}=\frac{2 \pi}{s\left(s-4 M^{2}\right)}\left(\left|\phi_{1}\right|^{2}+\left|\phi_{2}\right|^{2}+\left|\phi_{3}\right|^{2}+\left|\phi_{4}\right|^{2}+4\left|\phi_{5}\right|^{2}\right),
$$

while the total cross section is

$\sigma_{\mathrm{tot}}=\frac{4 \pi}{\sqrt{s} \sqrt{s-4 M^{2}}} \operatorname{Im}\left(\phi_{1}(s, 0)+\phi_{3}(s, 0)\right)$.

A general argument shows that $\phi_{4} \propto t, \phi_{5} \propto \sqrt{-t}$ as $t \rightarrow 0$, whereas $\phi_{1,2,3}$ go to a constant in this limit [40,41]. (Here we focus on the QCD part of the amplitude. The QED part behaves differently, see Appendix A.) Given these limiting behaviors, it is convenient to rescale the spin-dependent amplitudes as [24]

$r_{2}(s, t)=\frac{\phi_{2}(s, t)}{2 \operatorname{Im} \phi_{+}(s, t)}=R_{2}+i I_{2}$,

$r_{4}(s, t)=\frac{M^{2} \phi_{4}(s, t)}{-t \operatorname{Im} \phi_{+}(s, t)}=R_{4}+i I_{4}$,

$r_{5}(s, t)=\frac{M \phi_{5}(s, t)}{\sqrt{-t} \operatorname{Im} \phi_{+}(s, t)}=R_{5}+i I_{5}$,

where

$\phi_{+}(s, t) \equiv \frac{\phi_{1}(s, t)+\phi_{3}(s, t)}{2}$.

The complex functions $r_{i=2,4,5}$ have a finite limit as $t \rightarrow 0$. They can be experimentally accessed by measuring various spin asymmetries [24]. For example, $r_{5}$ is closely related to single spin asymmetry $A_{N}$, and $r_{2}$ is related to double spin asymmetry $A_{N N}$. The results from fixed-target experiments at RHIC at $\sqrt{s}=13.76 \mathrm{GeV}$ and $21.92 \mathrm{GeV}[42,43]$ indicate that the parameters $r_{2}, r_{5}$ are small, of order $10^{-3}$ in this lowenergy region. There are also RHIC data in the collider mode at $\sqrt{s}=200 \mathrm{GeV}[44,45]$. The analysis mostly focused on $A_{N}$ and a rather small value of $r_{5}$ has been reported.

At higher energies, however, nothing is known about the fate of the helicity-flip amplitudes since there is no polarized proton collider beyond the RHIC energies. They are rarely discussed in connection with the ongoing measurements at the LHC, or with the earlier measurements at the Tevatron. It is usually assumed, often without even mentioning it, that $\phi_{1} \approx \phi_{3}$ and $\phi_{2,4,5} \approx 0$ for all values of $t$. There is then only one (complex) amplitude $T=8 \pi \phi_{1}$, and (6) and (7) reduce to the formulas mentioned in the introduction. Yet, even in unpolarized scattering, the helicity-flip amplitudes affect the observables. In the presence of nonvanishing $\phi_{2}$, Eq. (2) should be modified as

$$
\left.\frac{d \sigma}{d t}\right|_{t=0}=\frac{\sigma_{\text {tot }}^{2}}{16 \pi}\left(1+\rho^{2}+2\left|r_{2}\right|^{2}\right) .
$$

Also, in the non-forward scattering with $|t|>0, \phi_{2,4,5}$ amplitudes could affect the shape of $d \sigma / d t$, especially, in the dip region where $\left|\phi_{1,3}\right|$ become small.

In the next section, we compute all the $\phi$ 's in a model which incorporates Pomeron and Odderon in the Wilson line formulation of small- $x$ QCD. We do not make the usual assumption that the helicity-flip amplitudes $\phi_{2,4,5}$ are negligibly small. As we shall see very clearly below, the spindependent Pomeron and Odderon exchanges naturally generate non-negligible helicity-flip amplitudes. The latter are a priori not suppressed at high energies since they share the same energy dependence ('Regge intercept') as for the helicity-conserving ones.

\section{Elastic scattering in the quark-diquark model}

In this section, we calculate the helicity amplitudes $\phi_{1, \ldots, 5}$ in the dipole model of high-energy $p p$ (and $p \bar{p}$ ) scattering illustrated in Fig. 1. Our setup is similar to the description of DIS at small- $x$ in the so-called 'dipole frame' where the virtual photon fluctuates into a quark-antiquark pair long before interacting with the target proton. Specifically, we work in an asymmetric frame in which $P_{1}^{+} \gg P_{2}^{-}$. The 'slow', leftmoving proton 2 is treated in the quark-diquark model [46]. It fluctuates into a quark and a scalar diquark, and the pair interacts with the shockwave created by the 'fast' proton 1 in the eikonal approximation. The corresponding scattering amplitude is given by 


$$
\begin{gathered}
T(s, t)=2 i s \int \frac{d^{2} r_{\perp}}{4 \pi} \int_{0}^{1} \frac{d z}{z(1-z)} \Psi^{*}\left(r_{\perp}, z, \lambda_{2}^{\prime}\right) \\
\times N\left(r_{\perp}, \Delta_{\perp}, \lambda_{1}, \lambda_{1}^{\prime}\right) \Psi\left(r_{\perp}, z, \lambda_{2}\right) .
\end{gathered}
$$

Here, $\Psi$ is the light-front wave function of the proton 2 fluctuation into a $q-q q$ pair to be specified shortly, $\lambda_{1,2}$ and $\lambda_{1,2}^{\prime}$ denote helicities of protons 1,2 in the initial and final states, respectively, $r_{\perp}$ is the transverse distance between the quark and the diquark, $z$ is the longitudinal momentum fraction of the proton 2 carried by the quark, and $N$ is the so-called dipole scattering amplitude defined by

$$
\begin{aligned}
& 2 P^{+} 2 \pi \delta\left(P^{+}-P^{\prime}\right) N\left(r_{\perp}, \Delta_{\perp}, \lambda_{1}, \lambda_{1}^{\prime}\right) \\
& \quad \equiv\left\langle P_{1}^{\prime}, \lambda_{1}^{\prime}\left|1-\frac{1}{N_{c}} \operatorname{Tr} U\left(r_{\perp} / 2\right) U^{\dagger}\left(-r_{\perp} / 2\right)\right| P_{1}, \lambda_{1}\right\rangle,
\end{aligned}
$$

in terms of a lightlike Wilson line in the fundamental representation

$U\left(x_{\perp}\right)=\mathrm{P} \exp \left(i g \int d z^{-} A^{+}\left(z^{-}, x_{\perp}\right)\right)$,

which describes the quark scattering off the target color field, and that for the diquark, $U^{\dagger}$, which has the same color representation as an antiquark. As usual, $g$ and $N_{c}=3$ denote the QCD coupling and number of colors.

Following [34], we parametrize the dipole amplitude as

$$
\begin{aligned}
\int d^{2} r_{\perp} e^{-i k_{\perp} \cdot r_{\perp}} N\left(r_{\perp}, \Delta_{\perp}, \lambda_{1}, \lambda_{1}^{\prime}\right) & (2 \pi)^{4} \delta^{(2)}\left(\Delta_{\perp}\right) \delta^{(2)}\left(k_{\perp}\right) \delta_{\lambda_{1}, \lambda_{1}^{\prime}} \\
= & \frac{g^{2}(2 \pi)^{3}}{8 N_{c} M\left(k_{\perp}^{2}-\Delta_{\perp}^{2} / 4\right)} \bar{u}\left(P_{1}^{\prime}, \lambda_{1}^{\prime}\right)\left\{\left[f_{1,1}+i \frac{k_{\perp} \cdot \Delta_{\perp}}{M^{2}} g_{1,1}\right]\right. \\
& +i \frac{\sigma^{i+}}{P_{1}^{+}} k_{\perp}^{i}\left[\frac{k_{\perp} \cdot \Delta_{\perp}}{M^{2}} f_{1,2}+i g_{1,2}\right] \\
& \left.+i \frac{\sigma^{i+}}{P_{1}^{+}} \Delta_{\perp}^{i}\left[f_{1,3}+i \frac{k_{\perp} \cdot \Delta_{\perp}}{M^{2}} g_{1,3}\right]\right\} u\left(P_{1}, \lambda_{1}\right)
\end{aligned}
$$

where the gluon GTMDS $f_{1, n}$ and $g_{1, n}(n=1,2,3)$ are functions of $k_{\perp}^{2}, \Delta_{\perp}^{2}$ and $\left|k_{\perp} \cdot \Delta_{\perp}\right|$ as well as the Bjorken- $x$ variable. At $\operatorname{small-} x$, they come from the real and imaginary parts of the operator $\operatorname{Tr} U U^{\dagger}$ and represent the Pomeron [47] and Odderon [48] exchanges, respectively. The apparent pole at $k_{\perp}^{2}=\Delta_{\perp}^{2} / 4$ is innocuous because $f$ and $g$ are proportional to $k_{\perp}^{2}-\Delta_{\perp}^{2} / 4$, see for example (36) below. Equation (14) describes the most general coupling between the Pomeron/Odderon and the proton, consistent with the symmetries of the GTMDs. By using the Gordon identity, one can write (14) as the linear combination of scalar and vector couplings. The tensor coupling [26] is absent in our framework.

Let us now work out the product of spinors explicitly. Up to corrections of order $M / P_{1}^{+}$and $\Delta_{\perp} / P_{1}^{+}$, we get

$$
\begin{gathered}
\bar{u}\left\{f_{1,1}+i \frac{k_{\perp} \cdot \Delta_{\perp}}{M^{2}} g_{1,1}+i \frac{\sigma^{i+}}{P_{1}^{+}} k_{\perp}^{i}\left[f_{1,2} \frac{k_{\perp} \cdot \Delta_{\perp}}{M^{2}}\right.\right. \\
\left.\left.\quad+i g_{1,2}\right]+i \frac{\sigma^{i+}}{P_{1}^{+}} \Delta_{\perp}^{i}\left[f_{1,3}+i \frac{k_{\perp} \cdot \Delta_{\perp}}{M^{2}} g_{1,3}\right]\right\} u \\
\approx 2 M \delta_{\lambda_{1}, \lambda_{1}^{\prime}}\left[f_{1,1}\left(k_{\perp}\right)+i \frac{k_{\perp} \cdot \Delta_{\perp}}{M^{2}} g_{1,1}\left(k_{\perp}\right)\right] \\
+2 \lambda_{1} \delta_{\lambda_{1},-\lambda_{1}^{\prime}} k_{\perp} \cdot \epsilon_{\lambda_{1}}\left[\frac{k_{\perp} \cdot \Delta_{\perp}}{M^{2}} f_{1,2}\left(k_{\perp}\right)+i g_{1,2}\left(k_{\perp}\right)\right] \\
+2 \lambda_{1} \delta_{\lambda_{1},-\lambda_{1}^{\prime}} \Delta_{\perp} \cdot \epsilon_{\lambda_{1}}\left[f_{1,3}\left(k_{\perp}\right)-\frac{1}{2} f_{1,1}\left(k_{\perp}\right)\right. \\
\left.+i \frac{k_{\perp} \cdot \Delta_{\perp}}{M^{2}}\left(g_{1,3}\left(k_{\perp}\right)-\frac{1}{2} g_{1,1}\left(k_{\perp}\right)\right)\right]
\end{gathered}
$$

where we introduced the 'polarization vector'

$\epsilon_{\lambda}=(1, i \lambda), \quad \Delta_{\perp} \cdot \epsilon_{\lambda}=\Delta_{\perp}^{1}+i \lambda \Delta_{\perp}^{2}=\sqrt{-t} e^{i \lambda \varphi}$.

In the $r_{\perp}$-space, the parametrization takes the form,

$$
\begin{aligned}
& N\left(r_{\perp}, \Delta_{\perp}, \lambda_{1}, \lambda_{1}^{\prime}\right)=(2 \pi)^{2} \delta^{(2)}\left(\Delta_{\perp}\right) \delta_{\lambda_{1}, \lambda_{1}^{\prime}} \\
& -\frac{g^{2}(2 \pi)^{3}}{4 N_{c} M}\left\{M \delta_{\lambda_{1}, \lambda_{1}^{\prime}}\left[\tilde{f}_{1,1}\left(r_{\perp}\right)+\frac{\Delta_{\perp} \cdot r_{\perp}}{M^{2} r_{\perp}^{2}} \tilde{g}_{1,1}\left(r_{\perp}\right)\right]\right. \\
& +\lambda_{1} \delta_{\lambda_{1},-\lambda_{1}^{\prime}} \frac{r_{\perp} \cdot \epsilon_{\lambda_{1}}}{r_{\perp}^{2}}\left[r_{\perp} \cdot \Delta_{\perp} \tilde{f}_{1,2}^{b}\left(r_{\perp}\right)+\tilde{g}_{1,2}\left(r_{\perp}\right)\right] \\
& +\lambda_{1} \delta_{\lambda_{1},-\lambda_{1}^{\prime}} \Delta_{\perp} \cdot \epsilon_{\lambda_{1}}\left[\tilde{f}_{1,2}^{a}\left(r_{\perp}\right)+\tilde{f}_{1,3}\left(r_{\perp}\right)\right. \\
& \left.\left.\left.-\frac{\tilde{f}_{1,1}\left(r_{\perp}\right)}{2}+\frac{\Delta_{\perp} \cdot r_{\perp}}{M^{2} r_{\perp}^{2}}\left(\tilde{g}_{1,3}\left(r_{\perp}\right)-\frac{\tilde{g}_{1,1}\left(r_{\perp}\right)}{2}\right)\right]\right\},(17)\right]
\end{aligned}
$$

with

$$
\begin{aligned}
& \tilde{f}_{1,1}\left(r_{\perp}\right)=\int \frac{d^{2} k_{\perp}}{(2 \pi)^{2}} e^{i k_{\perp} \cdot r_{\perp}} \frac{f_{1,1}\left(k_{\perp}\right)}{k_{\perp}^{2}-\Delta_{\perp}^{2} / 4}, \\
& \tilde{f}_{1,3}\left(r_{\perp}\right)=\int \frac{d^{2} k_{\perp}}{(2 \pi)^{2}} e^{i k_{\perp} \cdot r_{\perp}} \frac{f_{1,3}\left(k_{\perp}\right)}{k_{\perp}^{2}-\Delta_{\perp}^{2} / 4}, \\
& \tilde{f}_{1,2}^{a}\left(r_{\perp}\right)=\int \frac{d^{2} k_{\perp}}{(2 \pi)^{2}} e^{i k_{\perp} \cdot r_{\perp}}\left[\frac{k_{\perp}^{2}}{M^{2}}-\frac{\left(r_{\perp} \cdot k_{\perp}\right)^{2}}{r_{\perp}^{2} M^{2}}\right] \frac{f_{1,2}\left(k_{\perp}\right)}{k_{\perp}^{2}-\Delta_{\perp}^{2} / 4},
\end{aligned}
$$

$\tilde{f}_{1,2}^{b}\left(r_{\perp}\right)=\int \frac{d^{2} k_{\perp}}{(2 \pi)^{2}} e^{i k_{\perp} \cdot r_{\perp}}\left[2 \frac{\left(r_{\perp} \cdot k_{\perp}\right)^{2}}{r_{\perp}^{2} M^{2}}-\frac{k_{\perp}^{2}}{M^{2}}\right] \frac{f_{1,2}\left(k_{\perp}\right)}{k_{\perp}^{2}-\Delta_{\perp}^{2} / 4}$,

and

$\tilde{g}_{1, n}\left(r_{\perp}\right)=i \int \frac{d^{2} k_{\perp}}{(2 \pi)^{2}} e^{i k_{\perp} \cdot r_{\perp}} k_{\perp} \cdot r_{\perp} \frac{g_{1, n}\left(k_{\perp}\right)}{k_{\perp}^{2}-\Delta_{\perp}^{2} / 4}$,

for $n=1,2,3$. All functions defined above are real-valued.

The wave function of proton 2 in the quark-diquark model is given by (see "Appendix B" for the relevant Feynman rules) 


$$
\begin{gathered}
\Psi\left(r_{\perp}, z, \lambda_{2}\right)=-c_{s} \int \frac{d^{2} l_{\perp}}{(2 \pi)^{2}} e^{-i r_{\perp} \cdot l_{\perp}} \\
\times \frac{z(1-z) \bar{u}\left(z, l_{\perp}, \lambda_{q}\right) u\left(P_{2}, \lambda_{2}\right)}{l_{\perp}^{2}+\tilde{M}^{2}}
\end{gathered}
$$

where $c_{s}$ is a constant normalisation. The constituent quark has momentum fraction $z$, transverse momentum $l_{\perp}$, mass $m_{q}$ and helicity $\lambda_{q}$. The scalar diquark has momentum fraction $\bar{z}=1-z$ and mass $m_{s}$. Due to a finite binding energy, $m_{q}+m_{s} \geq M$, and this ensures that $\tilde{M}^{2} \equiv \bar{z} m_{q}^{2}+z m_{s}^{2}-$ $z \bar{z} M^{2} \geq 0$. After carrying out the integration over $l_{\perp}$, one can rewrite the wave function in Eq. (23) as,

$$
\begin{aligned}
& \Psi\left(r_{\perp}, z, \lambda_{2}\right) \\
& =\frac{-c_{s} \sqrt{z} \bar{z}}{2 \pi \sqrt{2}}\left[\delta_{\lambda_{q}, \lambda_{2}}\left(M z+m_{q}\right) K_{0}\left(\tilde{M}\left|r_{\perp}\right|\right)\right. \\
& \left.\quad-\delta_{\lambda_{q},-\lambda_{2}} \lambda_{2} r_{\perp} \cdot \epsilon_{\lambda_{2}}^{*} \frac{i \tilde{M}}{\left|r_{\perp}\right|} K_{1}\left(\tilde{M}\left|r_{\perp}\right|\right)\right] .
\end{aligned}
$$

This leads to the following expression for the wave function squared in the forward limit,

$$
\begin{aligned}
& \sum_{\lambda_{q}} \Psi\left(r_{\perp}, z, \lambda_{2}\right) \Psi^{*}\left(r_{\perp}, z, \lambda_{2}^{\prime}\right)=c_{s}^{2} \frac{z \bar{z}^{2}}{(2 \pi)^{2}}\left(\delta_{\lambda_{2}^{\prime}, \lambda_{2}} \frac{\Phi_{n}\left(r_{\perp}\right)}{M}\right. \\
& \left.\quad-2 i \lambda_{2} \delta_{\lambda_{2}^{\prime},-\lambda_{2}} r_{\perp} \cdot \epsilon_{\lambda_{2}}^{*} \Phi_{f}\left(r_{\perp}\right)\right),
\end{aligned}
$$

in terms of the helicity flip and helicity non-flip parts of the wave function

$$
\Phi_{n}\left(r_{\perp}\right)=M\left[\left(M z+m_{q}\right)^{2} K_{0}^{2}\left(\tilde{M}\left|r_{\perp}\right|\right)+\tilde{M}^{2} K_{1}^{2}\left(\tilde{M}\left|r_{\perp}\right|\right)\right]
$$

$\Phi_{f}\left(r_{\perp}\right)=\left(M z+m_{q}\right) K_{0}\left(\tilde{M}\left|r_{\perp}\right|\right) \frac{\tilde{M}}{\left|r_{\perp}\right|} K_{1}\left(\tilde{M}\left|r_{\perp}\right|\right)$,

respectively. In the non-forward case, a nontrivial phase $e^{i\left(z-\frac{1}{2}\right) \Delta_{\perp} \cdot r_{\perp}}$ emerges $^{3}$ [49]. We keep the subleading terms up to quadratic order in $\Delta_{\perp}$, so in practice we use

\footnotetext{
${ }^{3}$ For the reader's convenience, here we briefly recapitulate the discussion in Ref. [49]. The non-forward amplitude in dipole models typically has the following structure in impact parameter space $b_{\perp}$,
}

$$
T\left(b_{\perp}\right)=\int d^{2} \Delta_{\perp} e^{-i b_{\perp} \cdot \Delta_{\perp}} T\left(\Delta_{\perp}\right) \sim \int d^{2} r_{\perp}\left|\Psi\left(\Delta_{\perp}=0\right)\right|^{2} N\left(b_{\perp}-z r_{\perp}\right),
$$

where $N$ is the dipole scattering amplitude. The shift $b_{\perp} \rightarrow b_{\perp}-z r_{\perp}$ is caused by the phase factor $e^{i z r_{\perp} \cdot \Delta_{\perp}}$ which generically appears in non-forward impact factors [50], see Eq. (36) below for example. This implies that $b_{\perp}+(1-z) r_{\perp}$ and $b_{\perp}-z r_{\perp}$ can be interpreted as the coordinate of the quark and antiquark (or diquark), respectively. We can thus identify

$N\left(b_{\perp}-z r_{\perp}\right)=\left\langle 1-\frac{1}{N_{c}} \operatorname{Tr} U\left(b_{\perp}+(1-z) r_{\perp}\right) U^{\dagger}\left(b_{\perp}-z r_{\perp}\right)\right\rangle$.

$$
\begin{aligned}
& \sum_{\lambda_{q}} \Psi \Psi^{*} \rightarrow \sum_{\lambda_{q}} \Psi \Psi^{*} e^{i\left(z-\frac{1}{2}\right) \Delta_{\perp} \cdot r_{\perp}} \\
& \quad \approx \sum_{\lambda_{q}} \Psi \Psi^{*}\left[1+i z_{*} \Delta_{\perp} \cdot r_{\perp}-\frac{z_{*}^{2}\left(\Delta_{\perp} \cdot r_{\perp}\right)^{2}}{2}\right],
\end{aligned}
$$

where we used the abbreviation $z_{*}=z-1 / 2$. Assembling the above pieces together, we get

$$
\begin{aligned}
\int d^{2} r_{\perp} & \Psi^{*}\left(r_{\perp}, z, \lambda_{2}^{\prime}\right) N\left(r_{\perp}, \Delta_{\perp}, \lambda_{1}, \lambda_{1}^{\prime}\right) \Psi\left(r_{\perp}, z, \lambda_{2}\right) \\
= & -\frac{\pi g^{2} c_{s}^{2}}{2 N_{c} M} z \bar{z}^{2} \int d^{2} r_{\perp}\left\{\delta _ { \lambda _ { 2 } ^ { \prime } , \lambda _ { 2 } } \delta _ { \lambda _ { 1 } , \lambda _ { 1 } ^ { \prime } } \Phi _ { n } \left[\tilde{H}\left(1-\frac{z_{*}^{2}}{4} \Delta_{\perp}^{2} r_{\perp}^{2}\right)\right.\right. \\
& \left.-\delta^{(2)}\left(\Delta_{\perp}\right) \frac{2 N_{c}}{g^{2} \pi}+\frac{i z_{*} \Delta_{\perp}^{2}}{2 M^{2}} \tilde{g}_{1,1}\right] \\
& +\delta_{\lambda_{2}^{\prime},-\lambda_{2}} \delta_{\lambda_{1},-\lambda_{1}^{\prime}} \delta_{\lambda_{1}, \lambda_{2}} \Phi_{f} \\
& \times\left[-2 i \tilde{g}_{1,2}-i \frac{\Delta_{\perp}^{2}}{2 M^{2}}\left(2 \tilde{g}_{1,3}-\tilde{g}_{1,1}-z_{*}^{2} M^{2} r_{\perp}^{2} \tilde{g}_{1,2}\right)+\frac{z_{*} \Delta_{\perp}^{2} r_{\perp}^{2}}{2} \tilde{E}\right] \\
& -\delta_{\lambda_{2}^{\prime},-\lambda_{2}} \delta_{\lambda_{1},-\lambda_{1}^{\prime}} \delta_{\lambda_{1},-\lambda_{2}} \Phi_{f}\left(\Delta_{\perp} \cdot \epsilon_{\lambda_{1}}\right)^{2} \\
& \times\left[\frac{z_{*} r_{\perp}^{2}}{2} \tilde{E}-\frac{i}{2 M^{2}}\left(2 \tilde{g}_{1,3}-\tilde{g}_{1,1}-\frac{z_{*}^{2} M^{2} r_{\perp}^{2}}{2} \tilde{g}_{1,2}\right)\right] \\
& +\delta_{\lambda_{2}^{\prime}, \lambda_{2}} \delta_{\lambda_{1},-\lambda_{1}^{\prime}} \lambda_{1} \Delta_{\perp} \cdot \epsilon_{\lambda_{1}} \frac{\Phi_{n}}{2 M}\left[\tilde{E}+i z_{*} \tilde{g}_{1,2}\right] \\
& \left.+\delta_{\lambda_{2}^{\prime},-\lambda_{2}} \delta_{\lambda_{1}, \lambda_{1}^{\prime}} \lambda_{2} \Delta_{\perp} \cdot \epsilon_{\lambda_{2}}^{*} \Phi_{f}\left[z_{*} M r_{\perp}^{2} \tilde{H}-\frac{i \tilde{g}_{1,1}}{M}\right]\right\},
\end{aligned}
$$

where we defined

$\tilde{H} \equiv \tilde{f}_{1,1}=\int \frac{d^{2} k_{\perp}}{(2 \pi)^{2}} e^{i k_{\perp} \cdot r_{\perp}} \frac{f_{1,1}\left(k_{\perp}, \Delta_{\perp}\right)}{k_{\perp}^{2}-\Delta_{\perp}^{2} / 4}$,

and

$$
\begin{aligned}
\tilde{E} & \equiv 2 \tilde{f}_{1,3}-\tilde{f}_{1,1}+2 \tilde{f}_{1,2}^{a}+\tilde{f}_{1,2}^{b} \\
& =\int \frac{d^{2} k_{\perp}}{(2 \pi)^{2}} e^{i k_{\perp} \cdot r_{\perp}} \frac{-f_{1,1}\left(k_{\perp}\right)+2 f_{1,3}\left(k_{\perp}\right)+\frac{k_{\perp}^{2}}{M^{2}} f_{1,2}\left(k_{\perp}\right)}{k_{\perp}^{2}-\Delta_{\perp}^{2} / 4} \\
& \equiv \int \frac{d^{2} k_{\perp}}{(2 \pi)^{2}} e^{i k_{\perp} \cdot r_{\perp}} \frac{E\left(k_{\perp}, \Delta_{\perp}\right)}{k_{\perp}^{2}-\Delta_{\perp}^{2} / 4}
\end{aligned}
$$

Footnote 3 continued This gives

$$
\begin{aligned}
T\left(\Delta_{\perp}\right) \sim & \int d^{2} b_{\perp} e^{i b_{\perp} \cdot \Delta_{\perp}} \int d^{2} r_{\perp}|\Psi|^{2} \\
& \times\left\langle\operatorname{Tr} U\left(b_{\perp}+(1-z) r_{\perp}\right) U^{\dagger}\left(b_{\perp}-z r_{\perp}\right)\right\rangle \\
= & \int d^{2} b_{\perp} e^{i b_{\perp} \cdot \Delta_{\perp}} \int d^{2} r_{\perp} e^{i\left(z-\frac{1}{2}\right) \Delta_{\perp} \cdot r_{\perp}}|\Psi|^{2} \\
& \times\left\langle\operatorname{Tr} U\left(b_{\perp}+r_{\perp} / 2\right) U^{\dagger}\left(b_{\perp}-r_{\perp} / 2\right)\right\rangle .
\end{aligned}
$$


Above, $f_{1,1}$ and $E$ are nothing but the GTMD version of the GPDs of the fast proton (c.f., Eq. (4.48) of Ref. [51]) normalized as

$\int d^{2} k_{\perp} f_{1,1}\left(k_{\perp}, \Delta_{\perp}\right)=H(t), \quad \int d^{2} k_{\perp} E\left(k_{\perp}, \Delta_{\perp}\right)=E(t)$,

where $H$ and $E$ are the standard gluon GPDs. Note that, since we are colliding identical particles, by symmetry the coefficients of $\delta_{\lambda_{2}^{\prime},-\lambda_{2}} \delta_{\lambda_{1}, \lambda_{1}^{\prime}}$ and $\delta_{\lambda_{2}^{\prime}, \lambda_{2}} \delta_{\lambda_{1},-\lambda_{1}^{\prime}}$ have to be equal (up to a sign and trivial relabeling). However, in the asymmetric frame in which we are working, this is not obvious at first sight. While we do not have an explicit proof, we nevertheless argue that the two expressions are indeed equivalent. The functions $\Phi_{n}$ and $\Phi_{f}$ introduced in Eqs. (26) and (27) are related to the helicity non-flip and helicity flip parts of the gluon GTMD of the slow proton, respectively,

$$
\begin{aligned}
& G\left(k_{\perp}, \Delta_{\perp}\right) \propto C_{F} \alpha_{s}^{2} \int \frac{d z}{z \bar{z}} \int d^{2} r_{\perp} \\
& \quad \times \sum \Psi \Psi^{*} e^{i z \Delta_{\perp} \cdot r_{\perp}} \frac{k_{\perp}^{2}-\Delta_{\perp}^{2} / 4}{\left(\Delta_{\perp} / 2-k_{\perp}\right)^{2}\left(\Delta_{\perp} / 2+k_{\perp}\right)^{2}} \\
& \quad \times\left(1-e^{-i r_{\perp} \cdot\left(\Delta_{\perp} / 2-k_{\perp}\right)}\right)\left(1-e^{-i r_{\perp} \cdot\left(\Delta_{\perp} / 2+k_{\perp}\right)}\right) .
\end{aligned}
$$

The $t$-channel gluon propagators in Eq. (36) (as well as the small- $x$ evolution) are absorbed into $\tilde{E}$ and $\tilde{H}$. Thus, the terms proportional to $\Phi_{n} \tilde{E}$ and $\Phi_{f} \tilde{H}$ in the last two lines of Eq. (32) are both the convolution of the $H$-type GTMD of one proton and the $E$-type GTMD of the other proton, and are thus equal. A similar argument applies to the imaginary parts proportional to $\Phi_{n} \tilde{g}_{1,2}$ and $\Phi_{f} \tilde{g}_{1,1}$. Although there is in general no relation between $\tilde{g}_{1,2}$ and $\tilde{g}_{1,1}$, they satisfy the same evolution equation. The only difference is the way the $t$-channel Odderon amplitude $\mathcal{T}_{O}$ couples to the proton, and this coupling is proportional to $\Phi_{f}$ and $\Phi_{n}$, respectively, cf., Ref. [30]. Thus, the imaginary terms in the last two lines of Eq. (32) both have the structure $\Phi_{n} \otimes \mathcal{T}_{O} \otimes \Phi_{f}$, and are thus equivalent. After removing the phase according to Eq. (4), we arrive at

$$
\begin{aligned}
\phi_{1} & =\phi_{3}=-\frac{i s g^{2} c_{s}^{2}}{8 N_{c} M} \int_{0}^{1} d z \bar{z} \int \frac{d^{2} r_{\perp}}{4 \pi} \Phi_{n} \\
\times & {\left[\tilde{H}\left(1-\frac{z_{*}^{2}}{4} \Delta_{\perp}^{2} r_{\perp}^{2}\right)-\delta^{(2)}\left(\Delta_{\perp}\right) \frac{2 N_{c}}{g^{2} \pi}+\frac{i z_{*} \Delta_{\perp}^{2}}{2 M^{2}} \tilde{g}_{1,1}\right], } \\
\phi_{2} & =-\frac{s g^{2} c_{s}^{2}}{4 N_{c} M} \int_{0}^{1} d z \bar{z} \int \frac{d^{2} r_{\perp}}{4 \pi} \Phi_{f} \\
\times & {\left[\tilde{g}_{1,2}+\frac{\Delta_{\perp}^{2}}{4 M^{2}}\left(2 \tilde{g}_{1,3}-\tilde{g}_{1,1}-z_{*}^{2} M^{2} r_{\perp}^{2} \tilde{g}_{1,2}\right)+i \frac{z_{*}^{2}}{4} \Delta_{\perp}^{2} r_{\perp}^{2} \tilde{E}\right], }
\end{aligned}
$$

$$
\begin{aligned}
\phi_{4}= & \frac{i s g^{2} c_{s}^{2}}{16 N_{c} M}(-t) \int d z \bar{z} \int \frac{d^{2} r_{\perp}}{4 \pi} \Phi_{f}\left(z_{*} r_{\perp}^{2} \tilde{E}\right. \\
& \left.-\frac{i}{M^{2}}\left(2 \tilde{g}_{1,3}-\tilde{g}_{1,1}-\frac{z_{*}^{2} M^{2} r_{\perp}^{2}}{2} \tilde{g}_{1,2}\right)\right), \\
\phi_{5}= & \frac{i s g^{2} c_{s}^{2}}{16 N_{c} M} \sqrt{-t} \int_{0}^{1} d z \bar{z} \int \frac{d^{2} r_{\perp}}{4 \pi} z_{*} \\
& \times\left(2 \Phi_{f} M r_{\perp}^{2} \tilde{H}-\frac{i}{M} \Phi_{n} \tilde{g}_{1,2}\right) .
\end{aligned}
$$

The sign in front of $\tilde{g}_{1,2}$ in Eq. (40) has been fixed using the relation $\langle++|\tilde{T}|+-\rangle=-\langle++|\tilde{T}|-+\rangle[52]^{4}$. We immediately notice that $\phi_{1,3}$ are purely imaginary and $\phi_{2}$ is purely real. Therefore, the usual $\rho$-parameter (1) vanishes at $t=0$ in this model. Away from $t=0$, the $\rho$-parameter is dominated by the spin-independent Odderon $\tilde{g}_{1,1}$. We also see that the Pomeron $(\tilde{H}, \tilde{E})$ and Odderon $\left(\tilde{g}_{1,2,3}\right)$ contributions are always relatively imaginary. This means that there is no interference when squaring the amplitudes $\left|\phi_{i}\right|^{2}$, and $d \sigma / d t$ is insensitive to the sign of $\tilde{g}_{1,2,3}$. In other words, $d \sigma / d t$ is identical for $p p$ and $p \bar{p}$ scatterings in this model.

Recently, there are indications that the difference $d \sigma^{p p} / d t-d \sigma^{p \bar{p}} / d t$ is nonvanishing from an analysis of the LHC and Tevatron data [21,53]. In order to explain this, the Odderon has to have a small imaginary part (and the Pomeron has a small real part). It may be possible to generalize our model to accommodate this effect, for example, by using the dispersion relation or invoking Regge theory or the AdS/CFT correspondence [54]. This is however beyond the scope of this work.

As for the ratios (8), we get

$$
\begin{aligned}
& r_{2}(s, t=0)=\frac{\int d z \bar{z} \int d^{2} r_{\perp} \Phi_{f} \tilde{g}_{1,2}}{\int d z \bar{z} \int d^{2} r_{\perp} \Phi_{n}\left(\tilde{H}-\frac{\mathcal{A} N_{c}}{2 \pi^{3} g^{2}}\right)}=R_{2}+i I_{2} \\
& r_{4}(s, t \approx 0)=\frac{\int d z \bar{z} \int d^{2} r_{\perp} \Phi_{f}\left[-2 \tilde{g}_{1,3}+\tilde{g}_{1,1}+\frac{z_{*}^{2} M^{2} r_{\perp}^{2}}{2} \tilde{g}_{1,2}-i z_{*} M^{2} r_{\perp}^{2} \tilde{E}\right]}{2 \int d z \bar{z} \int d^{2} r_{\perp} \Phi_{n}\left(\tilde{H}-\frac{\mathcal{A} N_{c}}{2 \pi^{3} g^{2}}\right)} \\
& =R_{4}+i I_{4}, \\
& r_{5}(s, t \approx 0)=\frac{\int d z \bar{z} \int d^{2} r_{\perp} z_{*}\left[-\Phi_{n} \tilde{g}_{1,2}-2 i \Phi_{f} M^{2} r_{\perp}^{2} \tilde{H}\right]}{2 \int d z \bar{z} \int d^{2} r_{\perp} \Phi_{n}\left(\tilde{H}-\frac{\mathcal{A} N_{c}}{2 \pi^{3} g^{2}}\right)} \\
& =R_{5}+i I_{5} .
\end{aligned}
$$

Since $\phi_{4,5}$ vanish at $t=0, r_{4,5}$ are not well-defined at $t=0$, and of course measurements are always performed at $t \neq$ 0 . On the other hand, $r_{2}$ has a well-defined limit $t \rightarrow 0$, and there we need to subtract $(2 \pi)^{2} \delta^{2}\left(\Delta_{\perp}=0\right) \equiv \mathcal{A}$, the transverse area of the proton, from $\tilde{H}$ in the denominator. This converts the $S$-matrix $(\tilde{H})$ into the $T$-matrix, and is crucial for the $r_{\perp}$ integral to converge at small $r_{\perp}$. [Note

\footnotetext{
${ }^{4}$ Since the last two lines of Eq. (32) are equivalent as we have argued, we may choose any linear combination of $\{\tilde{H}, \tilde{E}\}$ and $\left\{\tilde{g}_{1,1}, \tilde{g}_{1,2}\right\}$ in Eq. (40). Here, we chose the set $\left\{\tilde{H}, \tilde{g}_{1,2}\right\}$ merely because we have numerical results available for these distributions.
} 
that $\Phi_{n, f}\left(r_{\perp}\right) \sim 1 / r_{\perp}^{2}$ at small- $r_{\perp}$.] When $t$ is nonzero, the subtraction is absent but there is no convergence problem since $\tilde{H}$ vanishes at $r_{\perp}=0$ if $\Delta_{\perp} \neq 0$. However, we can keep this subtraction in the denominator of $r_{4,5}$ and evaluate it at $t=0$ thanks to the fact that the $\operatorname{limit} \operatorname{Im} \phi_{1}(t \rightarrow 0)$ is smooth. Also, $\tilde{H}$ in the numerator of $r_{5}$ can be safely evaluated at $t=0$ since the factor $r_{\perp}^{2}$ kills the divergence at $r_{\perp}=0$. In the present work, these tricks are crucial for the numerical study in the next section since we do not have a numerical solution of $\tilde{H}$ at $t \neq 0$.

We see that the real parts $R_{2,4,5}$ entirely come from the Odderon. In particular, $R_{2}$ at $t=0$ is nonvanishing due to the spin-dependent Odderon $g_{1,2}$, and this can contribute to the differential and total cross section according to Eq. (10). The imaginary parts $I_{4,5}$ come from the Pomeron and $I_{2}$ vanishes in this model. It is interesting to notice that $I_{5}$ is parametrically of order unity if the typical value is $r_{\perp} \sim$ $1 / M$. However, at high energy the integrand is more localized at small- $r_{\perp}$, and then the factor $r_{\perp}^{2}$ leads to a suppression of $I_{5}$ (see below). We also expect $\left|I_{5}\right| \gg\left|I_{4}\right|$ assuming $|H| \gg|E|$.

\section{Energy dependence of the helicity amplitudes}

In this section, we study the center-of-mass energy $\sqrt{s}$ dependence of the helicity amplitudes $\phi_{i}$ and their ratios obtained in the previous section. $f_{1, n}$ and $g_{1, n}$ are the real and imaginary parts of the dipole scattering amplitude (12), respectively. The latter satisfies the Balitsky-Kovchegov (BK) equation $[47,55]$ which is an evolution equation in $\ln s$ including the gluon saturation effect. Thus, the Pomeron and Odderon amplitudes can be obtained from the real and imaginary parts of the BK equation with appropriate initial conditions $[48,56]$. We restrict ourselves to the forward limit $\Delta_{\perp}=0$, which means that we concentrate on $f_{1,1}$ and $g_{1,2}$. Solving the BK equation with finite $\Delta_{\perp}$ is numerically more involved, and to our knowledge this has not been done for the Odderon.

Admittedly, the use of the BK equation for our problem must be legitimately criticized. Being an equation originally derived in perturbation theory, in principle the BK equation can only apply to processes which involve a hard scale. However, in near-forward elastic $p p$ scattering, apparently there is no such hard scale. Yet, the idea of gluon saturation and the Color Glass Condensate [35] is that at asymptotically high energies, the gluon distribution in the colliding particles is characterized by a dynamically generated hard scale, called the saturation momentum $Q_{s}(s)$ which is an increasing function of $\sqrt{s}$. It has been demonstrated [57] that the theory of saturation with $Q_{s}^{2} \propto(\sqrt{s})^{0.23}>1 \mathrm{GeV}^{2}$ can successfully describe the multiplicity and the mean $p_{T}$ distribution in $p p$ collisions across the whole energy region of the LHC, $0.9 \mathrm{GeV}<\sqrt{s}<7 \mathrm{TeV}$. This partly justifies our approach at least for the Pomeron, and allows us to calculate the perturbative part of the growth of the total cross section with energy. Of course there are also nonperturbative contributions to the total cross section, but in our model these are absorbed into the parameter $\mathcal{A}$. As a matter of fact, the same argument does not quite hold for the Odderon. It has been noticed that the characteristic momentum scale of the Odderon amplitude does not grow like $Q_{s}[33,58]$. Therefore, the results involving Odderon below are at best a crude estimate of the possible high energy behavior suggested by perturbation theory. In reality the dominance of the nonperturbative effects may be overwhelming.

We basically follow Ref. [33] for the numerical evaluation of $f_{1,1}$ and $g_{1,2}$, except that we now include the running coupling effect. Reference [33] considered a transversely polarized proton and studied the gluon Sivers function which is the forward limit of $g_{1,2}$. On the other hand, in our problem the proton is longitudinally polarized. We thus need a little spinor algebra to connect the two works. Let us return to Eq. (14) and take the forward limit $\Delta_{\perp}=0$,

$$
\begin{gathered}
\int d^{2} r_{\perp} e^{-i k_{\perp} \cdot r_{\perp}} N\left(r_{\perp}\right)=(2 \pi)^{2} \delta^{(2)}\left(k_{\perp}\right) \mathcal{A} \\
-\frac{g^{2}(2 \pi)^{3}}{4 N_{c} M k_{\perp}^{2}}\left(M f_{1,1}+\epsilon^{i j} k_{\perp}^{i} S_{\perp}^{j} g_{1,2}\right) .
\end{gathered}
$$

Here we assume that the proton is transversely polarised, with the transverse spin vector $\vec{S}_{\perp}$ normalised as $\left|\vec{S}_{\perp}\right|=1$. In the $r_{\perp}$-space,

$N\left(r_{\perp}\right)=\mathcal{A}-\frac{g^{2}(2 \pi)^{3}}{4 N_{c} M}\left(M \tilde{H}\left(r_{\perp}\right)+i \frac{\epsilon^{i j} S_{\perp}^{i} r_{\perp}^{j}}{r_{\perp}^{2}} \tilde{g}_{1,2}\left(r_{\perp}\right)\right)$.

This can be written as (compare with Eq. (5) of [33])

$S\left(\vec{x}_{\perp}, \vec{y}_{\perp}\right)=P\left(r_{\perp}\right)+i \vec{S}_{\perp} \times \vec{r}_{\perp} Q\left(r_{\perp}\right), \quad \vec{r}_{\perp} \equiv \vec{x}_{\perp}-\vec{y}_{\perp}$,

where

$$
\begin{aligned}
& P\left(r_{\perp}\right)=\frac{g^{2}(2 \pi)^{3}}{4 N_{c} \mathcal{A}} \tilde{H}\left(r_{\perp}\right), \\
& Q\left(r_{\perp}\right)=\frac{g^{2}(2 \pi)^{3}}{4 N_{c} M \mathcal{A}} \frac{\tilde{g}_{1,2}\left(r_{\perp}\right)}{r_{\perp}^{2}},
\end{aligned}
$$

for the Pomeron and the spin-dependent Odderon components of the dipole $S$-matrix, respectively.

We compute $P\left(s, r_{\perp}\right)$ and $Q\left(s, r_{\perp}\right)$ as functions of the center-of-mass energy squared $s$ from the solution of the BK equation with running coupling as prescribed in Ref. [59]. Then, using Eq. (47) we access $\tilde{H}\left(r_{\perp}\right)$ and $\tilde{g}_{1,2}\left(r_{\perp}\right)$ that are further employed in computing $r_{2,5}$ through Eqs. (41) and (43). We adopt the following form for the coupling constant 


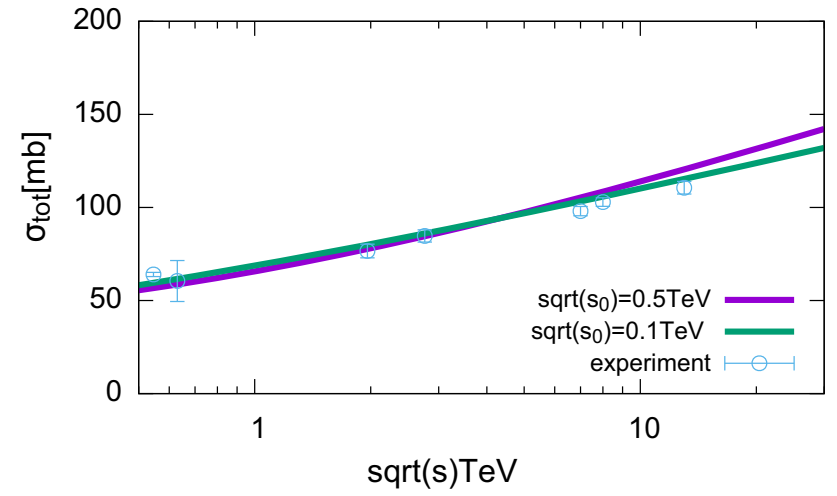

Fig. 2 The energy dependence of the total cross section. We take $Q_{s 0}=1.0 \mathrm{GeV}$ to fit the experimental data

$\alpha_{s}\left(r_{\perp}^{2}\right)=\frac{1}{b_{0} \log \left(\frac{4}{r_{\perp}^{2} \Lambda}+a\right)}$,

with $b_{0}=\frac{9}{4 \pi}$ (corresponding to $n_{f}=3$ ), $\Lambda=0.241 \mathrm{GeV}$ and $a=e^{\frac{8 \pi}{9}}$. The initial conditions are given at the starting energy scale $s_{0}$ as follows:

$P\left(s_{0}, r_{\perp}\right)=e^{-r_{\perp}^{2} Q_{s 0}^{2} / 4}, \quad Q\left(s_{0}, r_{\perp}\right)=\kappa Q_{s 0}^{3} r_{\perp}^{2} e^{-r_{\perp}^{2} Q_{s 0}^{2} / 4}$.

The initial saturation scale $Q_{s 0}$ is expected to be around 1 $\mathrm{GeV}$ in the $\mathrm{TeV}$ region, while the strength of Odderon $\kappa$ is an unknown parameter including its sign (see, however, [29]) which should be fitted to the data [34]. The other parameters in this model are $m_{q}, m_{s}$ and $c_{s}^{2} \mathcal{A}$ (only this product enters our observables). We fix $m_{q}=0.3 \mathrm{GeV}$ and $m_{s}=M-m_{q}$, while $c_{s}^{2} \mathcal{A}$ is fitted to the total cross section.

The energy dependence of the total cross section computed in our approach is shown in Fig. 2 with $Q_{s 0}=1.0$ $\mathrm{GeV}$. Here, the green and pink lines denote two different values of the starting energy scale, $\sqrt{s_{0}}=0.1$ and $0.5 \mathrm{TeV}$, respectively. The result is in reasonable agreement with the corresponding measurements in $p p$ collisions performed at several distinct energies, such as those by the TOTEM LHC Collaboration at $13 \mathrm{TeV}$ [12], $8 \mathrm{TeV}$ [60], $7 \mathrm{TeV}$ [61,62] and $2.76 \mathrm{TeV}$ [16], as well as in $p \bar{p}$ collisions by D0 Tevatron Collaboration at $1.96 \mathrm{TeV}$ [63] and by UA4 CERN SPS Collaboration at $546 \mathrm{GeV}$ [64] and $630 \mathrm{GeV}$ [65]. Since the measured values for $\sigma_{\text {tot }}(s)$ are sometimes not available in the experimental articles, in those cases the $\sigma_{\text {tot }}$ values have been taken from the global Lévy analysis of the corresponding elastic $p p$ and $p \bar{p}$ cross section data performed recently in Ref. [17]. Incidentally, we have also tried $Q_{s}=0.5 \mathrm{GeV}$, but the quality of the fit is noticeably worse in this case.

The results for $R_{2}, I_{5}$ and $R_{5}$ are plotted in Fig. 3 as functions of $\sqrt{s}$ in upper-left, upper-right and bottom panels, respectively. Note that the normalization and sign of $R_{2,5}$ are arbitrary, as it is proportional to the unknown parameter $\kappa$, and we have chosen $R_{5}$ to be negative following the recent suggestion in [66]. Irrespective of this, we can predict that $R_{2}$ and $R_{5}$ have the same sign and that $\left|R_{2}\right|$ is roughly two times larger than $\left|R_{5}\right|$. We also see a clear tendency that the magnitude of $R_{2,5}$ decreases with increasing energy. This is because, although the Odderon intercept is unity in the dilute (BFKL) regime, the nonlinear saturation effect tends to suppress the Odderon amplitude $[33,48,58]$. On the other hand, the value of $I_{5}$ is a prediction of this model, since both the denominator and numerator of (43) come from the Pomeron. It is negative and the magnitude decreases with energy because of the factor $r_{\perp}^{2}$ in the numerator of (43): The $r_{\perp}$-integral is dominated by $r_{\perp} \sim 1 / Q_{s}(s)$, and $Q_{s}(s)$ is an increasing function of energy.

The data on single spin asymmetry $A_{N}$ in small-angle elastic $p p$ collisions

$A_{N} \frac{d \sigma}{d t}=-\frac{4 \pi}{s\left(s-4 M^{2}\right)} \operatorname{Im}\left\{\left(\phi_{1}+\phi_{2}+\phi_{3}-\phi_{4}\right) \phi_{5}^{*}\right\}$

have recently become available from the fixed-target measurement HJET at BNL [67] as well as earlier from the STAR measurements of polarized elastic $p p$ collisions at $\sqrt{s}=200$ $\mathrm{GeV}$ [44]. These data have enabled to extract the real and imaginary parts of $r_{5}$ ratio in a wide energy domain. The values of $R_{5}$ published by the experimental collaborations were found (by STAR measurement and by an extrapolation from the lower HJET energies) to be either small positive or consistent with zero at $\sqrt{s}=200 \mathrm{GeV}$ (see also [26]), while the hadronic contribution predicted in Fig. 3 (upper-right panel) is found to be larger than the ballpark of experimental values.

We note, however, that the CNI contribution has to be taken into consideration as its impact on $r_{5}$ can be rather important, whereas the current analysis only focuses on the hadronic contribution to $\phi_{5}$. Indeed, as was recently advocated in Ref. [66] relying on a Regge analysis and a dominance of the Pomeron spin-flip contribution, the absorptive corrections to the Coulomb spin-flip amplitude significantly modify the CNI mechanism. As a result, this modification affects the extracted values of $r_{5}$, in particular making the spin-flip Pomeron $I_{5}$ rather large and negative, at the level of -5 to $-10 \%$ at $\sqrt{s}=200 \mathrm{GeV}$, in consistency with expectations [68]. The fact that our QCD-based approach predicts non-vanishing and negative $I_{5}$ is encouraging, although as we explained above it falls with energy, in contrast to the behavior predicted by the Regge fit of Ref. [66]. These results are not inconsistent and rather suggest that the gluon saturation regime has not been reached at RHIC energies. We leave a thorough analysis of the CNI effects in the current framework for a future work. 

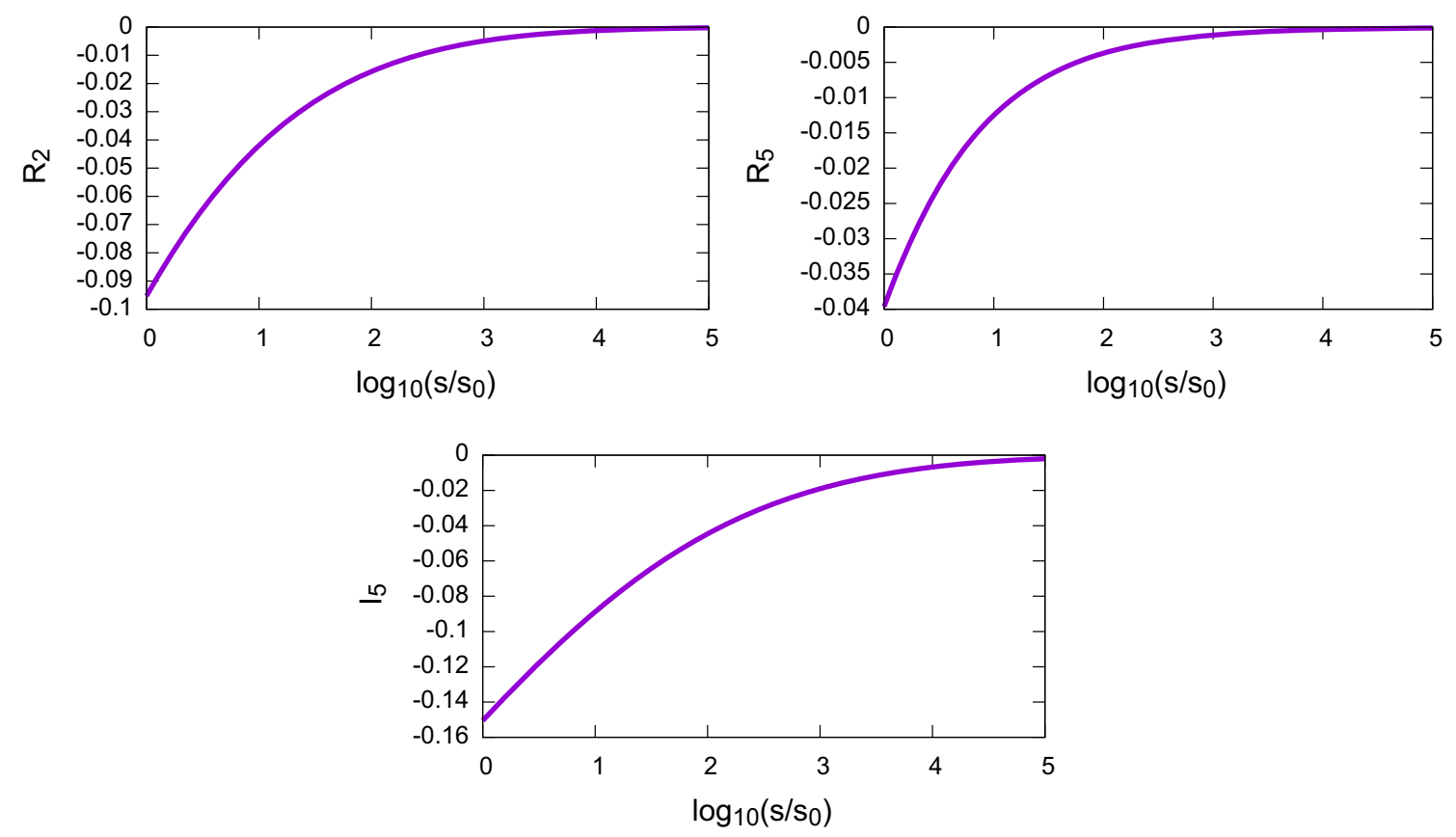

Fig. 3 The energy dependence of double-spin-flip $R_{2}$ (left) and single-spin-flip $r_{5}=R_{5}+i I_{5}$ (right and bottom) to non-flip ratios. Here, we take $\kappa=1 / 16$ and $Q_{s 0}=1.0 \mathrm{GeV}$

\section{Conclusions}

In this work, we have presented a new QCD-inspired model for small-angle elastic proton-(anti)proton scattering in terms of spin-dependent Pomeron and Odderon helicity amplitudes in the dipole picture based upon the Wilson line approach. The elastic amplitudes $\phi_{1, \ldots, 5}$ are effectively described in near-forward kinematics by means of a scattering of the lowest Fock state $p \rightarrow q+(q q)$ of projectile proton (i.e. the quark-diquark dipole) off the proton target, i.e. in a similar fashion as DIS. The corresponding dipole $S$-matrix receives contributions from non-flip and spin-flip Pomeron and Odderon exchanges that are represented in terms of GTMDs of different types.

Connecting to the numerical analysis of the small- $x$ Odderon evolution equation performed earlier in Ref. [33] and incorporating in addition the QCD running coupling effect, we explore the relative importance of spin-flip contributions to the elastic $p p$ scattering at high energies. In particular, we analyse the energy dependence of the spin-flip Pomeron $\left(I_{5}\right)$ and spin-flip Odderon $\left(R_{5}\right)$ amplitudes, as well as doublespin-flip Odderon $\left(R_{2}\right)$ amplitude relative to the non-flip one. At variance with an earlier Regge-based calculation of Ref. [66] incorporating for the first time the absorptive corrections in the CNI mechanism, we do not assume that the exchanged spin-independent and spin-dependent Regge trajectories have different intercepts and do not neglect the Odderon contributions. Yet, we have reached a qualitatively similar conclusion about a significant and negative contribu- tion to the single helicity-flip amplitude $I_{5}$. Moreover, the measured value of $R_{5}$ can be used to determine the Odderon coupling $\kappa$, which in turn determines the value of $R_{2}$. The energy dependence of $r_{5}$ in our approach is decaying and hence is strictly opposite to the steeply rising behavior from the Regge analysis [66] obtained in the lower energy region. This suggests that once the gluon saturation effect kicks in, the behavior of $r_{5}$ changes. A further analysis of this issue is certainly needed.

The experimentally probed energies in the existing measurements of the spin-flip contributions may not be high enough to make a conclusive statement about the energy dependence of spin-dependent Pomeron and, especially, Odderon effects. Indeed, at such low energies as $\sqrt{s}=200$ $\mathrm{GeV}$ the $C$-odd effects may come mostly from secondary Reggeon exchanges, not due to spin-dependent Odderon studied in our analysis here. It is therefore of high importance to perform a new measurement of $r_{2}$ and $r_{5}$ in a TeV energy range to make a definite conclusion about the energy dependence of spin-dependent Pomeron and Odderon in the future. Note that this does not necessarily require polarized proton beams which are not available at the LHC. The differential cross section (6) gets contributions from the helicity-flip amplitudes, but they are usually ignored in the CNI analysis. It would be very interesting to test more flexible parametrizations of the CNI effect including the hadronic and electromagnetic contributions to $\phi_{2,4,5}$. This could eventually affect the value of the $\rho$-parameter, and also the total cross section via (10). 
Finally, it is of course necessary to extend the present calculation to finite momentum transfer $t$, in particular up to the 'dip' region of $d \sigma / d t$. The basic formulas are given in (37)(40), but we are missing models of the spin-independent and spin-dependent Pomeron and Odderon amplitudes at finite $t$ (see for example $[69,70]$ for a model of $g_{1,1}$ at finite impact parameter). They can also serve as an initial condition for the impact-parameter dependent BK equation to determine the energy dependence. It is also interesting to consider different models for the 'slow' proton such as a bound state of three quarks. We hope to address these issues elsewhere.

Acknowledgements We thank Elliot Leader for useful correspondence. Y. H. thanks Shandong University in QingDao, where this collaboration started, for hospitality. R. P. is supported in part by the Swedish Research Council grants, contract numbers 621-2013-4287 and 201605996, by the Ministry of Education, Youth and Sports of the Czech Republic, project LTT17018, as well as by the European Research Council (ERC) under the European Union's Horizon 2020 research and innovation programme (grant agreement No. 668679). This work is supported by the U.S. Department of Energy, Office of Science, Office of Nuclear Physics, under contract No. DE- SC0012704, and in part by Laboratory Directed Research and Development (LDRD) funds from Brookhaven Science Associates. J. Zhou has been supported by the National Science Foundations of China under Grant No. 11675093, and by the Thousand Talents Plan for Young Professionals.

Data Availability Statement This manuscript has no associated data or the data will not be deposited. [Authors' comment: This is a theoretical work which does not contain any data to be deposited].

Open Access This article is licensed under a Creative Commons Attribution 4.0 International License, which permits use, sharing, adaptation, distribution and reproduction in any medium or format, as long as you give appropriate credit to the original author(s) and the source, provide a link to the Creative Commons licence, and indicate if changes were made. The images or other third party material in this article are included in the article's Creative Commons licence, unless indicated otherwise in a credit line to the material. If material is not included in the article's Creative Commons licence and your intended use is not permitted by statutory regulation or exceeds the permitted use, you will need to obtain permission directly from the copyright holder. To view a copy of this licence, visit http://creativecomm ons.org/licenses/by/4.0/.

Funded by SCOAP ${ }^{3}$.

\section{Appendix A: One photon exchange}

In this Appendix we quickly reproduce the helicity amplitudes in the one-photon exchange approximation. For a complete result, see [52]. The scattering amplitude is given by

$$
\begin{aligned}
i T= & -e^{2} \bar{u}\left(P_{3}, S_{3}\right)\left(\gamma^{\mu} F_{1}+\frac{i \sigma^{\mu \rho} \Delta_{\rho}}{2 M} F_{2}\right) u\left(P_{1}, S_{1}\right) \\
& \times \frac{-i}{t} \bar{u}\left(P_{4}, S_{4}\right)\left(\gamma_{\mu} F_{1}-\frac{i \sigma_{\mu \lambda} \Delta^{\lambda}}{2 M} F_{2}\right) u\left(P_{2}, S_{2}\right),
\end{aligned}
$$

where $\Delta=P_{3}-P_{1}=P_{2}-P_{4}$ and $F_{1}$ and $F_{2}$ are Dirac and Pauli form factors. This immediately gives

$T_{++++}=T_{+-+-}=8 \pi \phi_{1}=4 \pi \alpha_{e m} \frac{2 s}{t} F_{1}^{2}(t)$.

As for the double helicity-flip amplitudes, we use the formulas

$$
\begin{aligned}
& \bar{u}_{-\lambda}\left(P_{1}\right) \sigma^{+i} \Delta^{i} u_{\lambda}\left(P_{1}\right)=2 i P_{1}^{+} \lambda \Delta_{\perp} \cdot \epsilon_{\lambda}, \\
& \bar{u}_{-\lambda}\left(P_{2}\right) \sigma^{-i} \Delta^{i} u_{\lambda}\left(P_{2}\right)=2 i P_{2}^{-} \lambda \Delta_{\perp} \cdot \epsilon_{\lambda}^{*},
\end{aligned}
$$

to get

$$
\left\langle-\lambda,-\lambda^{\prime}|T| \lambda, \lambda^{\prime}\right\rangle \approx 4 \pi \alpha_{e m} \frac{s}{2 M^{2}} \frac{\lambda \lambda^{\prime} \Delta_{\perp} \cdot \epsilon_{\lambda} \Delta_{\perp} \cdot \epsilon_{\lambda^{\prime}}^{*}}{-t} F_{2}^{2} .
$$

We therefore find

$$
T_{--++}=8 \pi \phi_{2}=4 \pi \alpha_{e m} \frac{s}{2 M^{2}} F_{2}^{2} .
$$

For $\phi_{4,5}$, we need to remove the phase according to (4). The results are

$$
\begin{aligned}
& T_{+--+}=-4 \pi \alpha_{e m} \frac{s}{2 M^{2}} \frac{\left(\Delta_{\perp} \cdot \epsilon_{-}\right)^{2}}{-t} F_{2}^{2}, \\
& \phi_{4}=-\alpha_{e m} \frac{s}{4 M^{2}} F_{2}^{2}=-\phi_{2} . \\
& T_{+++-}=4 \pi \alpha_{e m} \frac{s}{M} \frac{\Delta_{\perp} \cdot \epsilon_{+}}{-t} F_{1} F_{2}, \\
& \phi_{5}=-\alpha_{e m} \frac{s}{2 M \sqrt{-t}} F_{1} F_{2} .
\end{aligned}
$$

\section{Appendix B: Feynman rules of the quark-diquark model}

In the diquark model [46], the interaction between the nucleon, the quark, and the scalar diquark is described by the following Feynman rules for the nucleon-quark-diquark vertex, quark-gluon vertex, and diquark-gluon vertex, respectively (see Fig. 4),

$$
i c_{s} \bar{u}\left(k, \lambda_{k}\right) u\left(P, S_{\perp}\right) \delta^{c c^{\prime}}, \quad-i g t^{a} \gamma^{\mu}, \quad-i g t^{a}\left(r+r^{\prime}\right)^{\mu},
$$

The scalar diquark, quark and gluon propagators in the Feynman gauge are given by

$$
\frac{i}{r^{2}-m_{s}^{2}+i \epsilon}, \quad \frac{i\left(k+m_{q}\right)}{k^{2}-m_{q}^{2}+i \epsilon}, \quad \frac{-i g^{\mu v} \delta^{c c^{\prime}}}{k^{2}+i \epsilon},
$$

where $c, c^{\prime}$ are color indices in the adjoint representation and $t^{a}$ are $\mathrm{SU}(\mathrm{N})$ gauge group generators in the fundamental representation. 


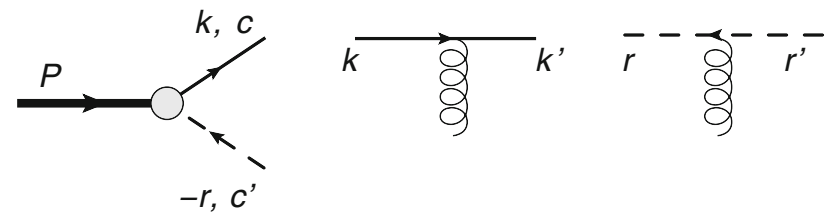

Fig. 4 Feynman rules of the quark-diquark model

\section{References}

1. I.M. Dremin, (2019). arXiv:1912.12841

2. J.R. Forshaw, D.A. Ross, Camb. Lect. Notes Phys. 9, 1 (1997)

3. A. Donnachie, P.V. Landshoff, Nucl. Phys. B 267, 690 (1986)

4. P. Collins, Cambridge Monographs on Mathematical Physics, An introduction to Regge theory and high-energy physics (Cambridge University Press, Cambridge, 2009)

5. J. Bartels, Nucl. Phys. B 175, 365 (1980)

6. J. Kwiecinski, M. Praszalowicz, Phys. Lett. 94B, 413 (1980)

7. J. Bartels, L.N. Lipatov, G.P. Vacca, Phys. Lett. B 477, 178 (2000). arXiv:hep-ph/9912423

8. L. Lukaszuk, B. Nicolescu, Lett. Nuovo Cim. 8, 405 (1973)

9. V.A. Khoze, A.D. Martin, M.G. Ryskin, Phys. Lett. B 780, 352 (2018). arXiv:1801.07065

10. E. Martynov, B. Nicolescu, Phys. Lett. B 786, 207 (2018a). arXiv:1804.10139

11. Y.M. Shabelski, A.G. Shuvaev, Eur. Phys. J. C 78, 497 (2018). arXiv: 1802.02812

12. G. Antchev et al., (TOTEM). Eur. Phys. J. C 79, 103 (2019a). arXiv: 1712.06153

13. G. Antchev et al. (TOTEM) (2018). arXiv:1812.08283

14. G. Antchev et al., (TOTEM). Eur. Phys. J. C 79, 785 (2019b). arXiv: 1812.04732

15. A. Ster, L. Jenkovszky, T. Csorgo, Phys. Rev. D 91, 074018 (2015). arXiv: 1501.03860

16. G. Antchev et al. (TOTEM) (2018). arXiv:1812.08610

17. T. Csörgő, R. Pasechnik, A. Ster, Eur. Phys. J. C 79, 62 (2019). arXiv: 1807.02897

18. V.A. Petrov, Eur. Phys. J. C 78, 221 (2018). arXiv:1801.01815 (Erratum: Eur. Phys. J. C 78(5), 414, 2018)

19. G. Pancheri, S. Pacetti, Y. Srivastava, Phys. Rev. D 99, 034014 (2019). arXiv: 1811.00499

20. E. Gotsman, E. Levin, I. Potashnikova, Phys. Lett. B 786, 472 (2018). arXiv: 1807.06459

21. T. Csörgő, T. Novak, R. Pasechnik, A. Ster, I. Szanyi (2019). arXiv: 1912.11968

22. M.G. Ryskin, Sov. J. Nucl. Phys. 46, 337 (1987)

23. M.G. Ryskin, Yad. Fiz. 46, 611 (1987)

24. N.H. Buttimore, B.Z. Kopeliovich, E. Leader, J. Soffer, T.L. Trueman, Phys. Rev. D 59, 114010 (1999). arXiv:hep-ph/9901339

25. E. Leader, T.L. Trueman, Phys. Rev. D 61, 077504 (2000). arXiv:hep-ph/9908221

26. C. Ewerz, P. Lebiedowicz, O. Nachtmann, A. Szczurek, Phys. Lett. B 763, 382 (2016). arXiv:1606.08067

27. S. Goloskokov, Phys. Rev. D 53, 5995 (1996). arXiv:hep-ph/9511382

28. S. Goloskokov, Phys. Lett. B 315, 459 (1993)

29. J. Zhou, Phys. Rev. D 89, 074050 (2014). arXiv: 1308.5912

30. L. Szymanowski, J. Zhou, Phys. Lett. B 760, 249 (2016). arXiv: 1604.03207

31. D. Boer, M.G. Echevarria, P. Mulders, J. Zhou, Phys. Rev. Lett. 116, 122001 (2016). arXiv: 1511.03485

32. H. Dong, D.-X. Zheng, J. Zhou, Phys. Lett. B 788, 401 (2019). arXiv:1805.09479
33. X. Yao, Y. Hagiwara, Y. Hatta, Phys. Lett. B 790, 361 (2019). arXiv: 1812.03959

34. R. Boussarie, Y. Hatta, L. Szymanowski, S. Wallon (2019). arXiv:1912.08182

35. F. Gelis, E. Iancu, J. Jalilian-Marian, R. Venugopalan, Ann. Rev. Nucl. Part. Sci. 60, 463 (2010). arXiv: 1002.0333

36. D.W. Sivers, Phys. Rev. D 41, 83 (1990)

37. M. Jacob, G.C. Wick, Ann. Phys. 7, 404 (1959)

38. M. Jacob, G.C. Wick, Ann. Phys. 281, 774 (2000)

39. M.L. Goldberger, M.T. Grisaru, S.W. MacDowell, D.Y. Wong, Phys. Rev. 120, 2250 (1960)

40. E. Leader, Camb. Monogr. Part. Phys. Nucl. Phys. Cosmol. 15, 1 (2011)

41. L.-L.C. Wang, Phys. Rev. 142, 1187 (1966)

42. I.G. Alekseev et al., Phys. Rev. D 79, 094014 (2009)

43. A.A. Poblaguev, Phys. Rev. D 100, 116017 (2019). arXiv: 1910.02563

44. L. Adamczyk et al., STAR. Phys. Lett. B 719, 62 (2013). arXiv: 1206.1928

45. L. Adamczyk, in Proceedings, 15th conference on Elastic and Diffractive scattering (EDS Blois 2013) (2013). arXiv:1311.3401

46. S.J. Brodsky, D.S. Hwang, B.-Q. Ma, I. Schmidt, Nucl. Phys. B 593, 311 (2001). arXiv:hep-th/0003082

47. I. Balitsky, Nucl. Phys. B 463, 99 (1996). arXiv:hep-ph/9509348

48. Y. Hatta, E. Iancu, K. Itakura, L. McLerran, Nucl. Phys. A 760, 172 (2005). arXiv:hep-ph/0501171

49. Y. Hatta, B.-W. Xiao, F. Yuan, Phys. Rev. D 95, 114026 (2017). arXiv: 1703.02085

50. J. Bartels, K.J. Golec-Biernat, K. Peters, Acta Phys. Polon. B 34 , 3051 (2003). arXiv:hep-ph/0301192

51. S. Meissner, A. Metz, M. Schlegel, JHEP 08, 056 (2009). arXiv:0906.5323

52. N.H. Buttimore, E. Gotsman, E. Leader, Phys. Rev. D 18, 694 (1978) (Erratum: Phys. Rev. D 35(1), 407, 1987)

53. E. Martynov, B. Nicolescu, Phys. Lett. B 778, 414 (2018). arXiv: 1711.03288

54. E. Avsar, Y. Hatta, T. Matsuo, JHEP 03, 037 (2010). arXiv:0912.3806

55. Y.V. Kovchegov, Phys. Rev. D 60, 034008 (1999). arXiv:hep-ph/9901281

56. Y.V. Kovchegov, L. Szymanowski, S. Wallon, Phys. Lett. B 586, 267 (2004). arXiv:hep-ph/0309281

57. L. McLerran, M. Praszalowicz, Acta Phys. Polon. B 41, 1917 (2010). arXiv:1006.4293

58. T. Lappi, A. Ramnath, K. Rummukainen, H. Weigert, Phys. Rev. D 94, 054014 (2016). arXiv:1606.00551

59. I. Balitsky, Phys. Rev. D 75, 014001 (2007). arXiv:hep-ph/0609105

60. G. Antchev et al., (TOTEM). Eur. Phys. J. C 76, 661 (2016). arXiv: 1610.00603

61. G. Antchev et al., TOTEM. EPL 101, 21004 (2013a)

62. G. Antchev et al., TOTEM. EPL 101, 21002 (2013b)

63. V.M. Abazov et al., D0. Phys. Rev. D 86, 012009 (2012). arXiv: 1206.0687

64. D. Bernard et al., UA4. Phys. Lett. B 198, 583 (1987)

65. D. Bernard et al., UA4. Phys. Lett. B 171, 142 (1986)

66. B.Z. Kopeliovich, M. Krelina (2019). arXiv:1910.04799

67. A.A. Poblaguev et al., Phys. Rev. Lett. 123, 162001 (2019). arXiv: 1909.11135

68. B.Z. Kopeliovich, B.G. Zakharov, Phys. Lett. B 226, 156 (1989)

69. A. Dumitru, G.A. Miller, R. Venugopalan, Phys. Rev. D 98, 094004 (2018). arXiv:1808.02501

70. A. Dumitru, V. Skokov, T. Stebel (2020). arXiv:2001.04516 